J3eA, Journal sur l'enseignement des sciences et technologies de l'information et des systèmes, Volume 4, Hors-Série 4, 1 (2005)

DOI : http://dx.doi.org/10.1051/bib-j3ea:2005851

(C) EDP Sciences, 2005

\title{
Contributions à la connaissance de la fiabilité des composants semiconducteurs de puissance
}

G. Coquery ${ }^{3}$, L. Dupont ${ }^{3,4}$, M. El Ghazouani ${ }^{1}$, F. Forest ${ }^{1}$, J.-J. Huselstein ${ }^{1}$, R. Jérisian ${ }^{2}$, Z. Khatir ${ }^{3}$, S. Lefebvre ${ }^{4}$, T. Lequeu ${ }^{2}$, S. Moreau ${ }^{2}$, F. Richardeau ${ }^{5}$, F. Saint-Eve ${ }^{4}$ et J. Vallon ${ }^{5}$

${ }^{1}$ LEM, Université Montpellier II

${ }^{2}$ LMP, Université de Tours - ST Microelectronics

${ }^{3}$ INRETS LTN Arcueil

${ }^{4}$ SATIE - ENS Cachan UMR

${ }^{5}$ LEEI ENSEEIHT - UMR INP Toulouse / CNRS 


\title{
COMMUNICATION DANS LES JOURNEES DU CLUB EEA
}

\author{
« Diagnostic en Electronique, Electrotechnique et Automatique »
}

\section{7-18 Mars 2005 Lyon}

\section{CONTRIBUTIONS A LA CONNAISSANCE DE LA FIABILITE DES COMPOSANTS SEMICONDUCTEURS DE PUISSANCE}

\begin{abstract}
(extrait du bilan scientifique à deux ans du GT Fiabilité - Diagnostic
\end{abstract} du GdR ME²-MS)

\begin{abstract}
Coquery G. ${ }^{(3)}$, Dupont L. ${ }^{(3,4)}$, El Ghazouani M. ${ }^{(1)}$, Forest $\mathbf{F}_{{ }^{(1)}}$, Huselstein J-J. ${ }_{(}^{(1)}$, Jérisian R. $^{(2)}$, Khatir Z ${ }^{(3)}$, Lefebvre S. ${ }^{(4)}$, Lequeu Th. ${ }^{(2)}$, Moreau $S^{(2)}$, Richardeau F. ${ }^{(5)}$, Saint-Eve F. ${ }^{(4)}$, Vallon J. ${ }^{(5)}$.

(1) LEM - Université Montpellier II, (2) LMP Université de Tours - ST Microelectronics, (3) INRETS LTN Arcueil, (4) SATIE - ENS Cachan UMR, (5) LEEI Enseeiht - UMR INP Toulouse / CNRS.
\end{abstract}

Cet article est issu du bilan de l'activité d'animation scientifique menée dans le cadre du groupe thématique ${ }^{\circ} 5$ du GdR ME²MS (Groupement de Recherche du CNRS sur la Maîtrise de l'Energie Electrique, des Matériaux aux Systèmes) depuis mars 2002, jusqu'à décembre 2003. Ce groupe a pour vocation de réunir les chercheurs des principaux Laboratoires impliqués dans le domaine de la Sûreté de Fonctionnement des dispositifs et des systèmes du Génie Electrique, en vue d'une activité d'échange et de collaboration.

Aujourd'hui, deux thématiques sont concernées par cette activité : la caractérisation de la fiabilité des semi-conducteurs de puissance sous contraintes, et le diagnostic de pannes des actionneurs électromécaniques. Cet article porte uniquement sur la première thématique où seront développés les méthodes d'études et les résultats. La seconde thématique fait l'objet d'une présentation spécifique (cf. programme des journées EEA - Diagnostic).

Résumé :

La diffusion et l'intégration des dispositifs d'électronique de puissance au sein de systèmes de plus en plus variés fait émerger aujourd'hui de nouvelles problématiques : la conception de technologies compatibles avec les nouvelles contraintes fonctionnelles et environnementales d'une part, et le développement de méthodes permettant de prédire le niveau de sûreté de ces dispositifs d'autre part. Dans ce contexte, la caractérisation de la fiabilité et la connaissance des mécanismes de défaillance sous contraintes, constitue un objectif incontournable pour qualifier et optimiser à terme de tels dispositifs. Sur cette base, l'article présente les principaux thèmes de recherche développés par les Laboratoires de Génie Electrique tant au niveau des composants actifs semi-conducteurs (régimes extrêmes répétitifs, fonctionnement à haute température) qu'au niveau du module de puissance (tenu au cyclage et aux chocs thermiques). Cet état des lieux montre bien les forces en présence aujourd'hui pour relever tous les défis posés par cette électronique de puissance diffusante. 


\section{1) RAPPEL DU CONTEXTE}

La sûreté de fonctionnement est un vaste domaine qui décrit, d'une façon générale, l'aptitude au fonctionnement sous contraintes, dans le temps et aux meilleures performances, des composants et des systèmes (fiabilité). L'aptitude à préserver une haute intégrité des composants et de leur environnement, en situation critique de défaillance, est une composante sous-jacente à la sûreté (sécurité).

La détection précoce de contraintes excessives (les causes) et l'analyse de leurs effets sur le vieillissement des constituants (diagnostic pré-défaillance) ainsi que les méthodes de surveillance - diagnostic post-défaillance contribuent, pour leur part, à une meilleure maintenabilité et disponibilité fonctionnelle.

Dans le contexte d'une très forte diffusion des dispositifs électriques au sein de systèmes de plus en plus variés et complexes, la maîtrise de la sûreté et de la sécurité de fonctionnement de ces dispositifs est devenu incontournable aujourd'hui dès la conception.

Transposée au Génie Electrique (matériaux, composants actifs, assemblage de puissance, convertisseur, actionneurs, process, réseaux, capteurs et commandes), la sûreté apparaît donc clairement comme une discipline transversale et pluridisciplinaire.

Aujourd'hui, il faut constater que les études de sûreté sont intimement liées à la pénétration croissante des systèmes électriques dans tous les secteurs industriels, de l'énergie et des transports. Cette tendance s'accompagne d'une grande diversité des contraintes (parfois nouvelles et combinées !) en nature (isolement, température, vibration, radiations, ...) et en amplitude (haute tension, haute température, cyclage actif pour la traction).

Une autre tendance forte est la volonté permanente de rechercher le meilleur compromis entre : a) le niveau de performance du système de plus en plus standardisé dans son architecture et dans ses technologies (compétitivité technico-économique), b) sa disponibilité opérationnelle (rentabilité économique), c) et la sécurité qu'il procure (relation homme machine ...). Ce constat permet de bien mesurer toute l'importance des études de sûreté mais également la grande diversité des problématiques qu'elle suscite. A ce titre, la figure 1, rappelle l'organisation d'une chaîne de conversion d'énergie électrique au niveau fonctionnel et technologique. 


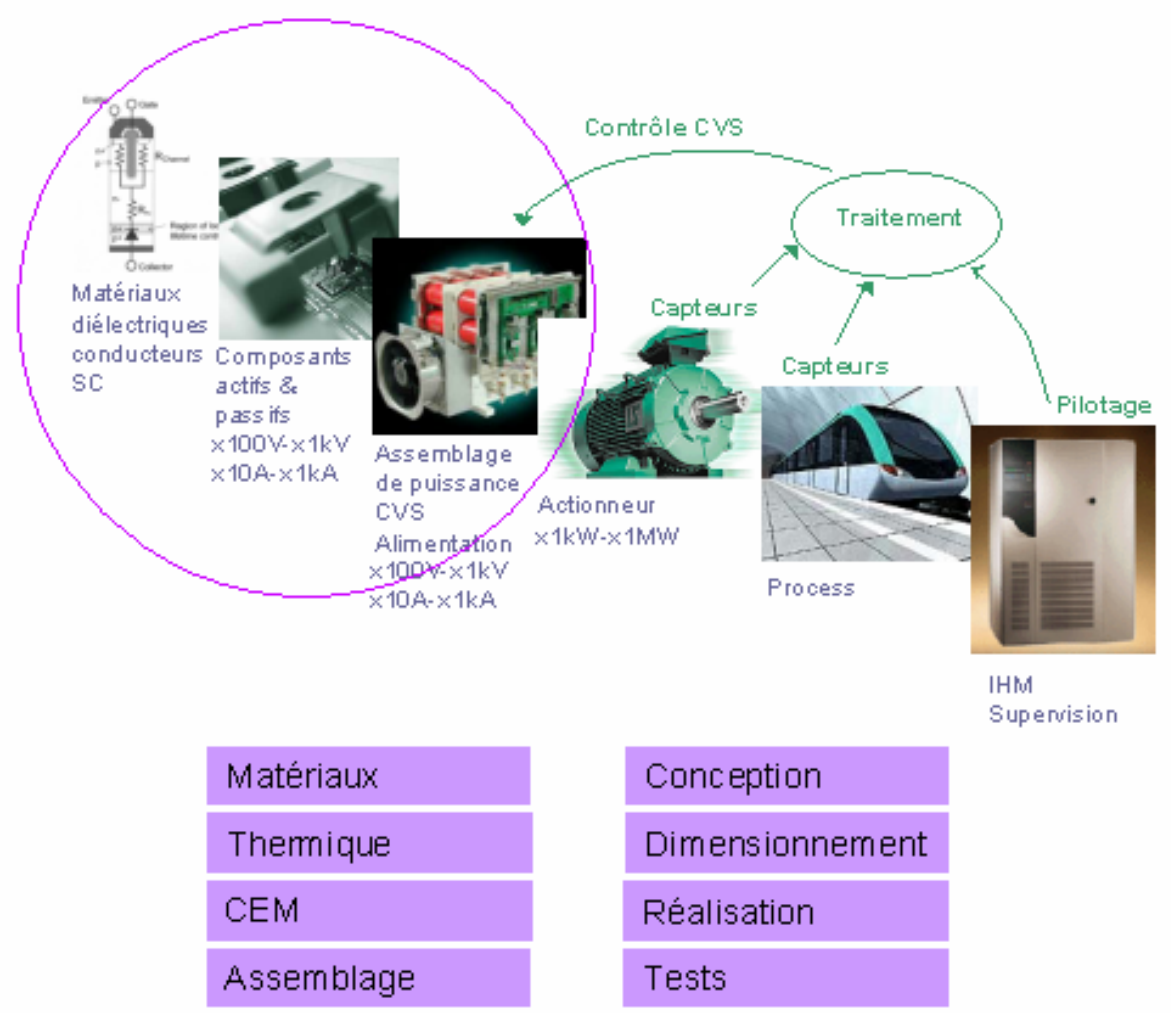

Figure 1. Organisation d'une chaîne de conversion d'énergie où apparaissent les dimensions technologique et méthodologique.

\section{2) OBJECTIFS}

Dans ce contexte et en restant pour le moment tout à fait général, trois objectifs scientifiques généraux peuvent être identifiés :

- l'identification et la maîtrise des contraintes principales. La liaison entre les contraintes et leurs effets, et inversement la liaison entre les points faibles (défauts intrinsèques, imperfections) et les contraintes appliquées. Cet objectif revient à décrire le composant ou le système à travers sa qualité, son vieillissement, i.e. sa fiabilité sous contraintes.

- le développement de méthodes et outils permettant un diagnostic pré/post défaillance.

- la conception de composants et d'architectures auto-protégés, reconfigurables, tolérantes aux défauts et intrinsèquement sûrs.

Néanmoins, il faut reconnaître que face à la diversité et à la complexité des phénomènes constituant des entraves à la fiabilité et indirectement à la sûreté des systèmes électriques, renforcé par le manque cruel de connaissances dans ce domaine (contrairement à d'autres comme la micro-électronique par exemple), les travaux menés par les Laboratoires se sont essentiellement portés sur l'analyse et la modélisation comportementale (expérimentale) d'une part ; et l'analyse physique des relations entre contraintes / limites physiques / défauts intrinsèques / imperfections technologiques, d'autre part. 
Sur cette base, mais de façon plus concrète cette fois, les recherches développées par les Laboratoires se sont organisées de la façon suivante, sous la forme de quatre projets :

- Projet 1 : INRETS/SATIE, durée de vie prévisionnelle et court-circuits répétitifs d'IGBT,

- Projet 2 : LEEI/LEM, suivi en nombre et en durée d'IGBT à haute température,

- Projet 3 : LMP/ST , chocs thermiques "silicium" par di/dt sur triac et "boîtier".

- Projet 4: INRETS cyclage actif sur modules à transistors IGBT.

La figure 2 illustre ainsi "la couverture scientifique" des projets, leur positionnement et leur complémentarité. La finalité commune de ces travaux pouvant également se définir par la recherche de la fiabilité prévisionnelle des composants de puissance sous contraintes réelles, le diagnostic pré-défaillance en situation de forte contrainte et enfin la définition de nouveaux composants mieux adaptés aux contraintes.

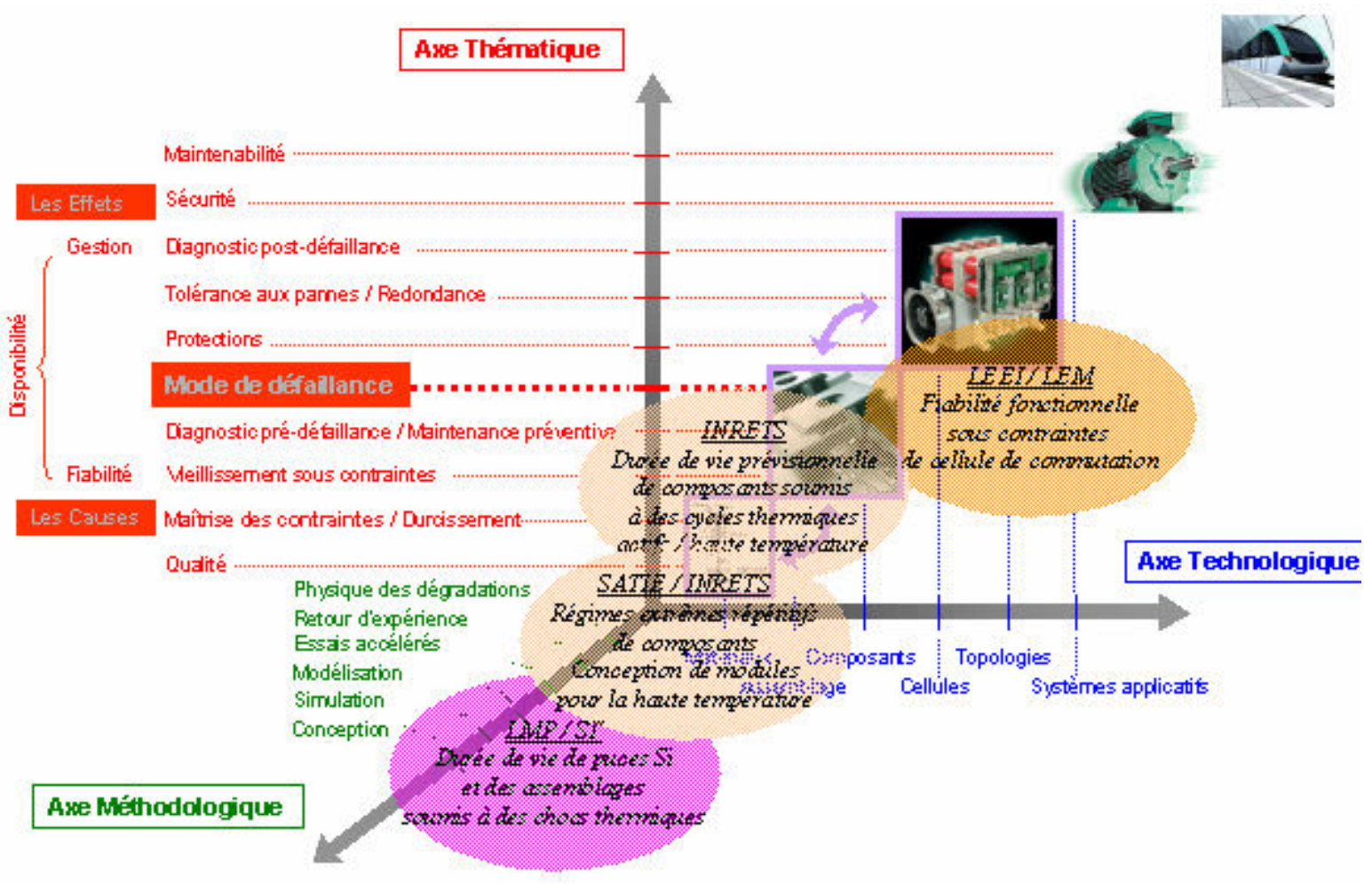

Figure 2. Positionnement et complémentarité des projets de recherche menés par les Laboratoires de Génie Electrique dans le domaine de la Fiabilité des composants de puissance.

\section{3) SYNTHESE DE L'ANIMATION SCIENTIFIQUE}

\section{1) Durée de vie prévisionnelle d'IGBT soumis à des court-circuit répétitifs}

Cette étude, pilotée par le Laboratoire Satie, porte sur la caractérisation de transistors de puissance MOSFET et IGBT (Insulated Gate Bipolar Transistor) soumis à des régimes extrêmes répétitifs et dans des conditions d'énergie contrôlée. Les régimes extrêmes sont le court-circuit (non saturation du transistor sous la pleine tension d'alimentation) et l'avalanche. 
L'objectif étant, encore une fois, de relier l'intensité de la contrainte (amplitude et durée) aux mécanismes impliqués dans la dégradation physique du composant jusqu'à sa défaillance. Les retombées peuvent se traduire par la définition de composants nouveaux durcis et par une meilleure connaissance des limites réelles des composants actuels.

Dans les travaux présentés, un premier banc de test de court-circuits a permis de caractériser des composants IGBT 600/1200V 20/30A PT/NPT et CoolMos 600V/20A selon des courbes Ncycles $=\mathrm{f}$ (Energie de contrainte) pour deux températures de boîtier : $25^{\circ} \mathrm{C}$, $125^{\circ} \mathrm{C}$. Des cycles de différentes énergies sont appliqués sur une période de $3 \mathrm{~s}$ jusqu'à destruction du composant.

Comme le montre la figure 3 , les courbes révèlent une énergie critique (ou densité d'énergie critique) au delà de laquelle la défaillance de la puce se produit en un cycle seulement et en deçà de laquelle la puce tient un très grand nombre de cycles $(>10000)$. Bien que le mode défaillance soit unique (fusion localisée de la puce), il semble que différents mécanismes de défaillance soient impliqués dans ce processus extrême selon que la défaillance complète se produise durant la phase statique (génération thermique du cristal), au blocage (focalisation par champ local - densité de courant locale) ou à l'état bloqué (emballement par courant de fuite). Ces mécanismes semblent par ailleurs dépendants de la technologie du composant testé ce qui rend l'étude encore plus délicate.

En parallèle, des simulations physiques électro-thermiques par éléments finis ont permis d'étayer l'analyse expérimentale, en particulier sur la connaissance de la température max locale résultant de la contrainte. Une tentative de suivi des caractéristiques électriques (dérives et vieillissement éventuels) a été initiée également, mais aujourd'hui il semble qu'aucun "signe" physique précurseur à la défaillance ne soit accessible [1].
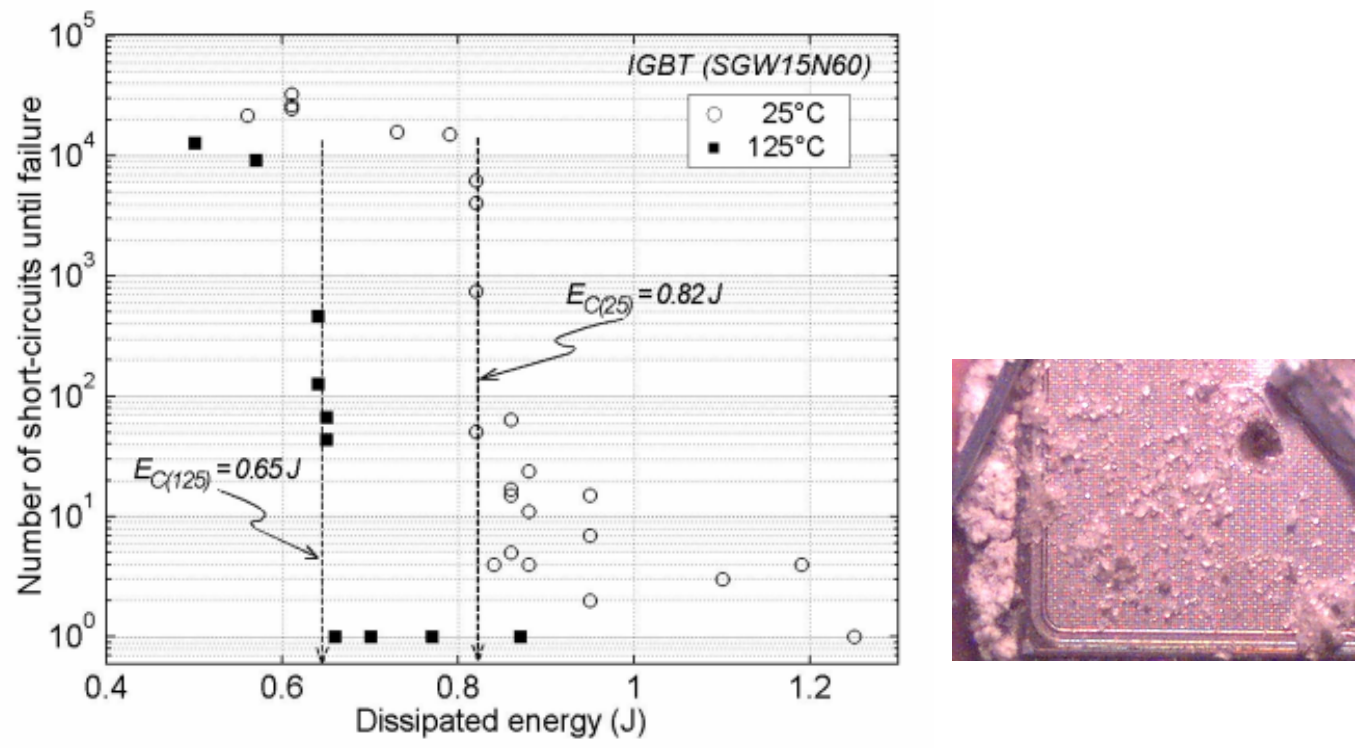

Figure 3. Bilan expérimental mettant en relation l'énergie appliquée sur une puce de transistor IGBT $600 \mathrm{~V} / 15 \mathrm{~A}$ et le nombre de cycles avant la défaillance physique. La photo montre le résultat d'une focalisation de conduction suivie de la fusion locale de la puce. 


\section{2) Banc de contraintes pour cellules de commutation à haute température}

Ce projet implique le LEEI et le LEM sur une étude de fiabilité d'un ensemble d'onduleurs fonctionnant en régime continu non cyclé et à haute température. L'étude porte sur l'ensemble des constituants d'un convertisseur élémentaire (modules IGBT, drivers, condensateurs de filtrage, alimentations auxiliaires). Contrairement aux projets 1 et 3 , il s'agit donc d'une approche beaucoup plus macroscopique qui consiste à mener des essais en nombre et en durée pour suivre l'évolution des caractéristiques (en absolu et en dispersion) sur l'ensemble des composants de puissance. Un premier banc, dont une photo est donnée en figure 4, a été conçu et mis en exploitation en février 2002 et comprend deux ponts complets à IGBT $50 \mathrm{~A} / 600 \mathrm{~V}$ utilisés à $\mathrm{Tj}=150^{\circ} \mathrm{C}$. Chaque pont est constitué par la mise en opposition de deux modules (bras) au travers d'une simple self $45 \mu \mathrm{H}$. L'ensemble est piloté par une modulation numérique rapide (modulation différentielle inter-bras).

L'intérêt d'une telle architecture est de pouvoir mener des essais en durée avec comme alimentation une puissance alignée sur les pertes totales seulement. Un dispositif de protection rapproché permet de confiner l'apparition d'un premier défaut au sein d'un bras. Un système d'acquisition permet de contrôler et d'enregistrer en continu les grandeurs BF principales (un échantillonnage rapide est prévu sur quelques périodes de modulation précédent un défaut sur la puissance). Le banc totalise aujourd'hui plus de $3500 \mathrm{~h}$ de fonctionnement et a fait l'objet de deux campagnes de caractérisation : caractérisations statiques sur les IGBT en relation avec le LAAS, caractérisation dynamique au LEEI et caractérisation des condensateurs au LEM. Aujourd'hui, seules les mesures réalisées sur les condensateurs ont permis de révéler un processus de vieillissement des condensateurs (augmentation de la résistance série par évaporation et altération de l'électrolyte). Ce vieillissement a été assez marqué sur la première tranche $1500 \mathrm{~h}$ (de 10\% à 30\%) puis s'est ralenti dans la deuxième tranche. Ce vieillissement apparaît, par ailleurs, de plus en plus inhomogène dans le temps (problème de qualité et de conditions d'emploi différentes). Une thèse a été soutenue sur le sujet en décembre 2003 [2], une seconde thèse est en cours au LEM.

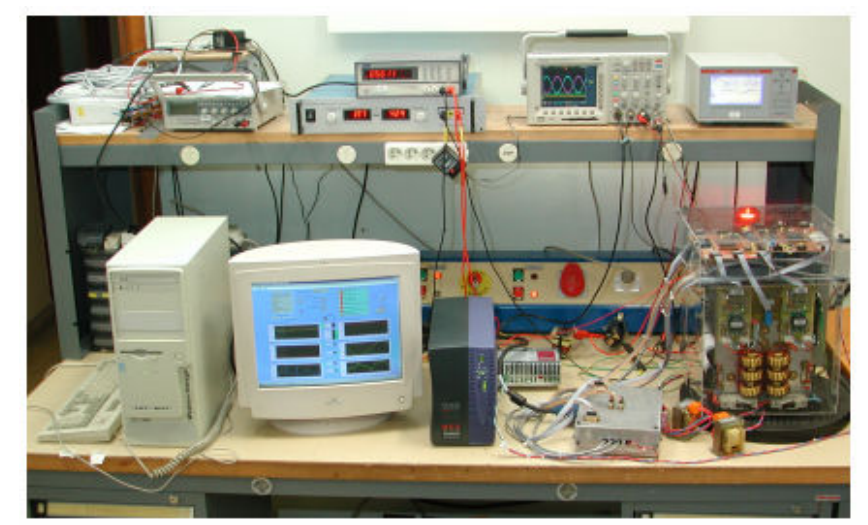

Figure 4. Plate-forme de contraintes "continues" électrique et thermique pour cellules de commutation à modules de puissance IGBT.

\section{3) Chocs thermiques "silicium" par di/dt et chocs thermiques sur boîtier.}

Ce projet implique le LMP de l'université de Tours et le laboratoire de STMicroelectronic Tours sur des problématiques de chocs thermiques répétitifs pratiqués, soit au niveau de la puce, soit au niveau du boîtier [3]. 
Les chocs "puce" correspondent assez typiquement à un phénomène de focalisation lors de la fermeture d'un composant Triac avec son snubber parallèle. Si le di/dt lors de cette phase est trop fort, la dilatation locale de la puce peut produire une micro-fissure visible au travers du courant de fuite excessif. Un banc de test complet a donc été réalisé par le LMP de façon à caractériser la durée de vie de Triacs soumis à un fort di/dt selon une courbe Ncycles = $\mathrm{f}(\mathrm{di} / \mathrm{dt})$. Une modélisation par lois de Weibull a permis d'une part d'extrapoler les résultats aux conditions nominales et d'autre part de révéler le mode de défaillance (qualité ou vieillissement). Les résultats montrent qu'il s'agit davantage d'un problème de qualité (présence de lacunes au sein du cristal) et non de vieillissement. Une analyse physique et technologique couplée à de la simulation par éléments finis a permis également de localiser la contrainte mécanique (volumique ou surfacique) en fonction de l'amplitude de la contrainte et du quadrant d'allumage du Triac. Cette démarche très complète est encore une fois tout à fait exemplaire pour la communauté.

Les chocs thermiques "boîtier" constituent aujourd'hui la poursuite des travaux du LMP toujours en relation étroite avec les moyens du laboratoire d'analyse des défaillances de ST. Il s'agit ici d'évaluer la qualité et la robustesse de boîtiers TO220 ainsi que l'assemblage interne au moyen de chocs liquide-liquide $-55^{\circ} \mathrm{C} / 125^{\circ} \mathrm{C}$. La dégradation constatée des composants se manifeste par la propagation d'une fissure dans le joint substrat-socle-boîter. La démarche consiste ici à suivre l'évolution de la résistance thermique d'un lot de composants en fonction du nombre de chocs. Le critère de fin de vie étant une augmentation de $20 \%$ de la résistance thermique. Une modélisation statistique par loi de Weibull est ensuite réalisée.

Parallèlement, une simulation thermo-mécanique par éléments finis sous Ansys permet, sur la base du même assemblage, de déterminer l'énergie de déformation relative à la même contrainte expérimentale. Cette connaissance permet d'identifier les paramètres d'une loi générique de fatigue (loi de Darveaux) Ncycles $=\mathrm{f}(\Delta \mathrm{W})$. Cette loi doit permettre de relier, par simulation interposée, la contrainte thermique appliquée au composant à sa durée de vie prévisionnelle. Ceci nécessite la mesure des constantes mécaniques des brasures à l'aide d'un DMA et d'un TMA (mesures extrêmement longues et coûteuses). Là encore il faut noter l'intérêt d'une telle démarche à la fois en termes de modélisation mais aussi en termes de connaissances physiques. Cette démarche est d'ailleurs assez courante dans le domaine des micro-assemblages et tend naturellement à apparaître dans le domaine de la puissance. 

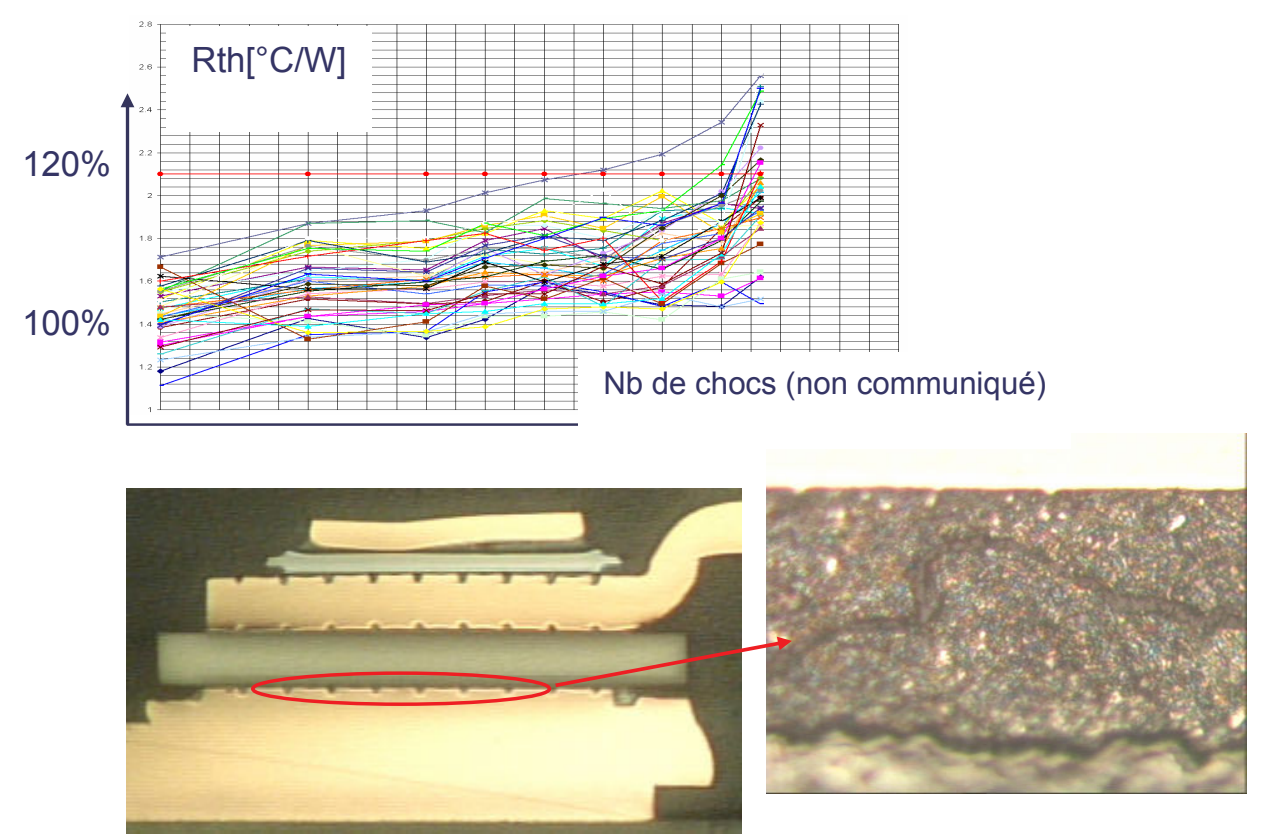

Figure 5. Coupe d'un assemblage de puissance d'un boîtier TO220. Mise en évidence d'une fissuration du joint de brasure. Suivi de la résistance thermique de l'assemblage d'une population de composants

\section{4) Cyclage thermique par auto-échauffement de modules IGBT}

Cette étude est pilotée par le LTN de l'INRETS (Laboratoire des Technologies Nouvelles), connue est reconnue pour son expertise expérimentale dans le domaine du cyclage actif de puissance de composants IGBT employés pour la traction ferroviaire et plus récemment pour la traction sur véhicule hybride (composant 200/400A 600V entre autres).

Ces composants utilisent des puces Si déposées sur un substrat isolant métallisé (DBC), lui même déposé sur un socle $\mathrm{Cu}$ ou AlSiC et d'une liaison filaire par faisceaux de bondings au niveau des métallisations de surface de la puce. La contrainte thermique cyclée se traduit par des contraintes mécaniques au niveau du joint brasé substrat-socle (délamination par cyclage lent de qqs dizaines de secondes) ainsi qu'au niveau des pieds de bondings (microfissures par cyclage rapide de l'ordre de la seconde).

Le premier domaine d'études consiste donc à caractériser, par essais "accélérés", la durée de vie de ce type d'assemblage selon un réseau de courbes Ncycles $=\mathrm{f}(\Delta \mathrm{T} j, \Delta \mathrm{T}$ case $)$ et en relation avec des critères de défaillance préalablement identifiés comme "sensibles". Le second domaine d'études porte sur l'exploitation de ce réseau de référence pour évaluer de façon prévisionnelle la durée de vie d'un module en situation "nominale" de cyclage. Les retombées attendues se situent au niveau d'une maintenance prédictive "pré-défaillance", et dans le choix de la technologie la mieux adaptée.

Cette démarche complète a été explorée sur la base d'un véhicule hybride urbain. Les essais accélérés pratiqués en laboratoire ont porté sur du cyclage lent / rapide avec $40^{\circ} \mathrm{C}<$ $\Delta \mathrm{Tj}, \Delta \mathrm{T}$ case $<80^{\circ} \mathrm{C}$ et se traduisent par une durée de vie de quelques milliers de cycles à plusieurs centaines de milliers. Des loi exponentielles extrapolées ont permis de modéliser cette phase expérimentale. Les indicateurs de fin de vie sont Vcesat $(+5 \%)$ en cyclage rapide et Rthj-c(+20\%) en cyclage lent. La figure 6 donne à ce titre un exemple de lois extrapolées 
par rapport à du cyclage rapide en $\Delta \mathrm{Tj}$ (puce) et en cyclage lent en $\Delta \mathrm{Tc}$ (boîtier). Parallèlement une modélisation du profil de mission "type" des modules a été établie sous la forme d'une courbe de distribution des cycles en fonction de l'ondulation de température. Cette modélisation a été obtenue par une mesure directe de la température de la puce au moyen d'une fibre optique (à terme, un estimateur de température sera implanté). La difficulté consiste ensuite à corréler la courbe de distribution aux courbes de laboratoire pour savoir si le nombre de cycles de "fin de vie" est atteint ou non. Cette corrélation, ou plus exactement, cette normalisation des courbes est réalisée en faisant l'hypothèse que le processus d'endommagement est proportionnel au nombre de cycles à contrainte constante, hypothèse sujette à beaucoup de discussions actuellement de la part des spécialistes.

Quoiqu'il en soit, cette démarche a permis de réduire la courbe de distribution de cycles en un point équivalent cumulé normalisé à $\Delta \mathrm{Tj}=40^{\circ} \mathrm{C}$ et $\Delta \mathrm{T}$ case $=40^{\circ} \mathrm{C}$. Pour ces deux points, on constate que le nombre de cycles normé est bien inférieur au nombre de cycles issu du régime accéléré selon le critère de fin de vie retenu. Une confrontation expérimentale plus large doit bien sûr confirmer toutes les hypothèses posées dans la démarche. Mais au delà de cette tentative, c'est bien la démarche globale assez exemplaire qu'il faut retenir et souligner [4].

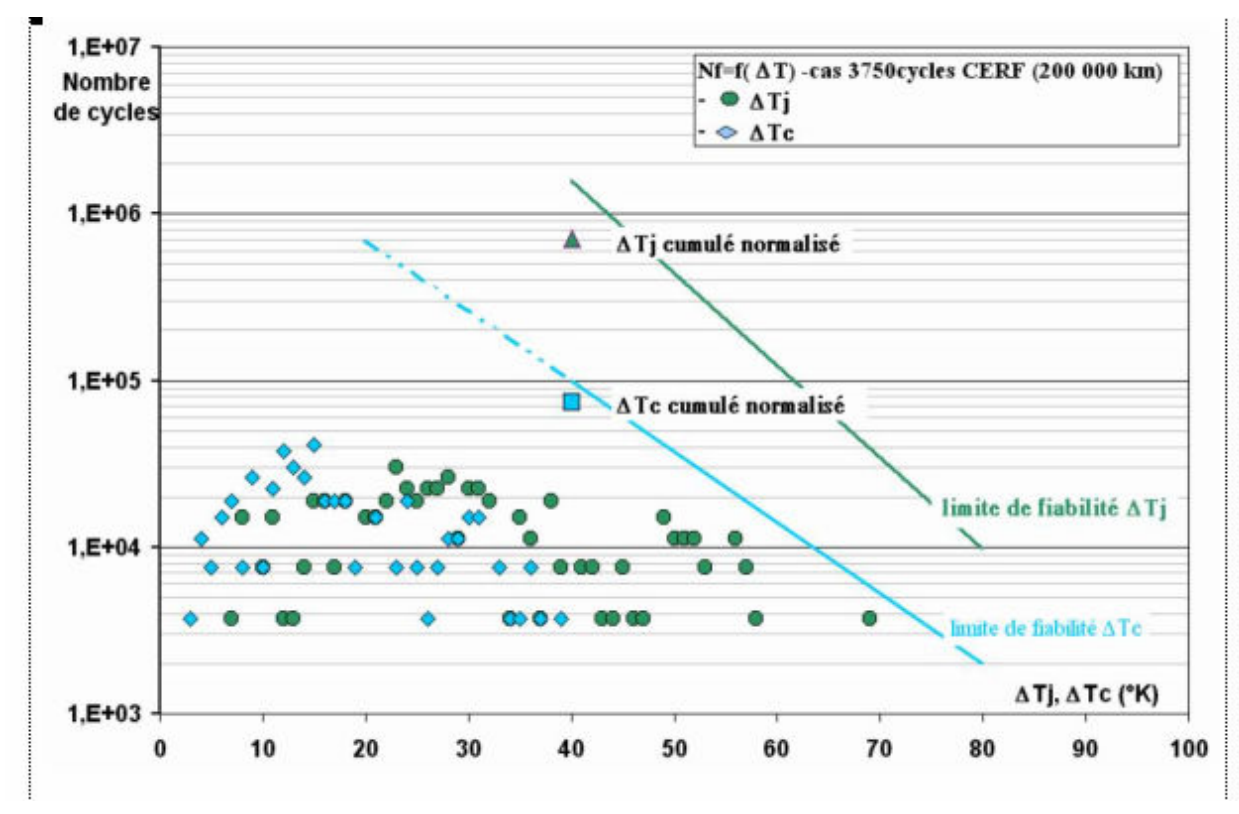

Figure 6. Normalisation et corrélation d'un nuage de points en ( $\Delta \mathrm{T}-\mathrm{Ncyles})$ issus d'un profil de mission réel par rapport à des lois de fatigues extrapolées issues d'essais accélérés en Laboratoire.

\section{4) Conclusion}

La forte pénétration des composants semi-conducteurs d'électronique de puissance au sein de systèmes de plus en plus variés fait émerger aujourd'hui de nouvelles problématiques : la conception de nouveaux assemblages, le développement des technologies compatibles avec les nouvelles contraintes fonctionnelles (profils de mission) et environnementales (température) d'une part, le développement de méthodes permettant de prédire le niveau de performance de ces dispositifs dans le temps d'autre part. Dans ce contexte, la caractérisation de la fiabilité et de la durée de vie de ces composants associée à la connaissance des mécanismes de défaillance sous contraintes, constituent un objectif incontournable pour qualifier et optimiser à terme de tels dispositifs. Sur cette base, cet article a tenté d'exposer les 
principaux axes de recherche développés par les Laboratoires de Génie Electrique tant au niveau des composants actifs semi-conducteurs (régimes extrêmes répétitifs, fonctionnement à haute température) qu'au niveau du module de puissance (tenu au cyclage et aux chocs thermiques). Cet état des lieux montre bien les forces en présence aujourd'hui pour relever tous les défis posés par cette électronique de puissance de plus en plus diffusante.

\section{5) Bibliographie}

[1] "Influence des régimes extrêmes de fonctionnement sur la durée de vie des composants semi-conducteurs de puissance", thèse de l'ENS de Cachan, soutenue par Frédéric Saint-Eve en décembre 2003 au Laboratoire Satie.

[2] "Introduction à l'étude de la fiabilité des cellules de commutation à transistors IGBT soumis à de fortes contraintes", thèse de l'INP Toulouse soutenue par Jérôme Vallon le 17 décembre 2003 à l'ENSEEIHT.

[3] "Etude de la fiabilité comparative de triac entre cyclages thermiques et chocs thermiques", Stéphane Moreau, Thierry Lequeu et Robert Jérisian, Congrès Electronique de Puissance du Futur, Toulouse, septembre 2004.

[4] "Contribution à l'identification et à l'estimation des contraintes de fatigue thermique des convertisseurs intégrés pour la traction électrique", Stéphane Carubelli, thèse de l'Université Henri Poincaré, Nancy I, LTN-INRETS, 25 septembre 2003. 